\title{
RASGOS ESENCIALES DE LA RED
}

\section{ESSENTIAL FEATURES OF NETWORK}

\section{AUTOR/ES}

Beatriz Peña Acuña: Profesora contratada doctora de la Facultad de Ciencias Sociales y de la Comunicación. Universidad San Antonio. Murcia (España).

pietrafelice@hotmail.com

\section{RESUMEN}

Esta disertación es un comentario acerca de dos conocidos artículos: “Características de la comunicación en Red" de López García y "Lecciones de la Historia de Internet" de Manuel Castells (2001). El escrito pretende animar al lector a la lectura del artículo con contenido vigente. Este ensayo se muestra como comentario crítico que puede guiar sobre los aspectos más brillantes y novedosos que proponen estos dos artículos sobre el mundo de Internet.

\section{PALABRAS CLAVE}

Red - Internet - ARPANET - Hipertextualidad - Interactividad.

\section{ABSTRACT}

This dissertation is a commentary on two popular items: "Characteristics of Network" by William Lopez Garcia and "Communication Lessons from the History of the Internet" by Manuel Castells (2001). The document aims to encourage the reader to read the article with current content. This essay shows how that can guide critical commentary on the most brilliant and innovative two articles on the Internet world.

\section{KEY WORDS}

Network - Internet - ARPANET - Hypertext - Interactivity. 
ÍNDICE
$\boldsymbol{\sim}$ INTRODUCCIÓN
$\boldsymbol{\sim}$ 1. CARACTERÍSTICAS DE LA COMUNICACIÓN EN RED
$\boldsymbol{\sim}$ INTRODUCCIÓN
1.1 Ideas fundamentales del artículo
1.2 Estructura del artículo
주 1.2.1 Consistencia del discurso
줄 1.2.2 Relación lógica entre ideas
ح 2. LECCIONES DE LA HISTORIA DE INTERNET
$\boldsymbol{\sim}$ INTRODUCCIÓN
2.1 Ideas fundamentales de este artículo
2.2 Estructura
$\sim$ CONCLUSIONES
^ BIBLIOGRAFÍA RECOMENDADA

\section{INTRODUCCIÓN}

Esta disertación es un comentario acerca de dos conocidos artículos: Características de la comunicación en Red y Lecciones de la Historia de Internet. El escrito pretende animar al lector a la lectura del artículo con contenido vigente. Este ensayo se muestra como comentario crítico que puede guiar sobre los aspectos más brillantes y novedosos que proponen estos dos artículos sobre el mundo de Internet. Asimismo realiza una crítica de su redacción para que sirva de orientación al lector.

\section{CARACTERÍSTICAS DE LA COMUNICACIÓN EN RED}

\section{INTRODUCCIÓN}

Este artículo me ha aportado con claridad el concepto de comunicación en red y de un formato más evolucionado que el HTML, el XML. Me ha parecido sugerente explicar la distinta cobertura y uso de los medios tradicionales y los medios de la comunicación en la red para entender la diferencia entre ellos.

Desde un punto de vista crítico, cuando el autor dice que se están poniendo dificultades para el desarrollo de la hipertextualidad en los medios digitales me parece un poco exagerado. Se trata de una evolución de mentalidad en la producción por parte de creativos que todavía han trabajado en medios tradicionales. Sin embargo, las nuevas generaciones de jóvenes con una mentalidad más internauta seguramente desarrollaran más esta posibilidad. 


\subsection{Ideas fundamentales del artículo}

Establece la cronología de nacimiento de la revolución comunicativa en los años 90 con la aparición de Internet. A través de esta revolución comunicativa se ha dado el crecimiento económico de EE.UU. Además de la revolución comunicativa confluyen otras dos revoluciones tecnológicas: la informática y la digital.

Entre los beneficios de la revolución digital frente al formato anterior analógico se cuentan: la mejor calidad, almacenaje, reproducción, copia y ahorro de tiempo y espacio. Los contenidos digitales favorecen un nuevo modelo de comunicación llamado "comunicación en red". Esta comunicación en red contempla cuatro características: la ruptura de tiempo y espacio, el carácter multimedia, la hipertextualidad y la interactividad.

La ruptura de tiempo procura una ventaja espacial y temporal sobre los medios tradicionales. Además posee unas ventajas que le son propias como la instantaneidad, la ruptura de la periodicidad, la universalidad, la capacidad de almacenamiento.

El carácter multimedia procura un nuevo lenguaje multimedia en el que se combinan formatos diferentes como el texto escrito, el sonido, las imágenes, las bases de datos, los programas autoejecutables y los elementos interactivos. El lenguaje multimedia viene facilitado por el código HTML.

Todavía falta mentalidad para explotar las potencialidades del discurso multimedia debido a limitaciones de tipo económico, tecnológico y cultural.

El discurso multimedia tiene la dificultad que es todavía demasiado novedoso, pues tiene reglas distintas al medio escrito o al audiovisual.

La hipertextualidad abre la posibilidad al usuario a una experiencia comunicativa abierta, a que personalice y seleccione con libertad en la red.

El hipertexto procura una estructuración novedosa de la información, pues se interconecta con ideas relacionadas al modo humano de discurrir. Se diferencia de los medios tradicionales que exigen del usuario una lectura secuencial.

El usuario tiene un mayor poder de decisión sobre la información que quiere consumir y el orden de lectura que quiere conceder a los contenidos. Se trata de un modo de lectura personalizado.

Las páginas web tienen la cualidad de que se actualizan, su dinamismo consiste en un cambio y reescritura continua. 
La construcción de sentido no sólo la otorga el productor sino también el receptor. Es un medio más contextual que los anteriores.

La estructura abierta del hipertexto permite diversos recorridos de lectura, una estructura constantemente redefinida y una estructura informativa que trasciende el propio medio.

No parece que la mayor parte de los contenidos presentes en Internet desarrollen del todo las potencialidades del hipertexto debido a que sigue vigente una mentalidad que tiene en cuenta la estructura de la información de los medios tradicionales. Pese a todo el hipertexto se va desarrollando. Debido a esta mentalidad, se pierde la posibilidad de tener acceso a opciones infinitas, a la creatividad productiva, y al enlace con fuentes externas.

La interactividad tiene una triple proyección: la interactividad con el emisor, con la información y con otros usuarios. La interactividad con el emisor y en el caso del plano privado supone que los medios digitales facilitan unos medios para que se pueda comunicar con los consumidores. En los medios digitales los espacios dedicados a la interactividad son más numerosos que en los medios tradicionales.

La interactividad con la información supone la posibilidad de navegar a través de un abanico de opciones y el establecimiento de filtros y preferencias. El navegante se convierte en un emisor de contenidos en el caso de las weblogs. En el caso de los medios tradicionales, donde se da una mayor personalización a través de un menú personalizado es en las plataformas digitales de televisión. Gracias al lenguaje HTML y sobre todo del XML se calcula un mayor desarrollo. La interactividad con otros usuarios en los medios digitales se potencia sin comparación a través de múltiples medios como los chats, foros, grupos de noticias, tablones de anuncios, juegos en red, formularios, encuestas, correo electrónico y programas de transferencia de archivos entre usuarios.

En definitiva, la comunicación en red abre unas expectativas diferentes que los medios tradicionales.

\subsection{Estructura del artículo}

\subsubsection{Consistencia del discurso}

El discurso hilvanado por el autor es consistente, porque ha argumentado bien las afirmaciones. Además ha tratado de ser claro, poner títulos y componer la argumentación en base a ellos y a las clasificaciones internas o apartados. 
Existe, sin embargo, algún error. Primero, el título 2.1. (De la sociedad industrial a la sociedad del conocimiento) es una introducción en realidad al apartado siguiente 2.2, que tendría que ser 2.1.2 porque forma parte de 2.1. El segundo y tercer error lo realiza cuando podría haber titulado a partir del segundo párrafo de la página 39 con "Ventajas de los medios digitales", por ejemplo, y en el segundo párrafo también de la página 44 "Problemas con los medios tradicionales".

\subsubsection{Relación lógica entre ideas}

El redactor muestra por lo general coherencia en el discurso pero también tiene varios errores. El segundo párrafo de la página 38 no es nada claro por la alusión que pone en la mitad del párrafo sobre el cambio social, podría haberlo omitido porque además no lo desarrolla. Esta afirmación interrumpe la lógica de las preguntas que formula al principio y que desarrolla en el tercer párrafo. Además en el cuarto párrafo enlaza el concepto bits con el concepto binario del quinto párrafo sin ninguna aclaración de lo que es cada uno de ellos.

En la página 39 segundo párrafo cita a Walter Benjamín y es una cita un tanto postiza no añade ni aclara nada. De hecho pone más citas que nos han parecido redundantes como la de la página 45 de Kerckhove y la de Castells. Acude con demasiada frecuencia a autores para definir conceptos claros como la secuencialidad en la página 50. O expone citas con ideas obvias (en el primer párrafo de la página 56 cita de Álvarez).

\section{LECCIONES DE LA HISTORIA DE INTERNET}

\section{INTRODUCCIÓN}

Este artículo es interesante por la perspectiva histórica del fenómeno mediático nuevo: Internet. No conocía la historia del proyecto de Internet y me han parecido apasionantes todos los pasos que tuvieron que dar para llegar al proyecto actual. Sobre todo, porque muchos investigadores que lo promovieron tenían una motivación sana de crear algo nuevo y útil, incluso muchos eran todavía estudiantes o estudiantes de doctorado. Me ha gustado mucho la colaboración cooperativa que se establece entre los nodos de las universidades.

Me ha parecido inteligente la táctica del Departamento de Defensa para ganar la batalla a su homólogo ruso: fomentar la creatividad entre los suyos para ganarle la batalla tecnológica militar. 
Me ha parecido que es un artículo documentado y en el que el autor ha investigado mucho. Por ejemplo, se ha molestado en averiguar qué nivel de investigación tecnológica militar tenían los rusos.

\subsection{Ideas fundamentales de este artículo}

Internet es un proyecto que muestra la capacidad de las personas para trascender las reglas institucionales, superar las barreras burocráticas y subvertir los valores convencionales para crear un nuevo instrumento revolucionario.

El presente artículo pretende descubrir las lecciones que subyacen en la creación de Internet que surge de la confluencia de muchas sinergias públicas y privadas, en el ámbito internacional y en varios campos interdisciplinares.

En cuanto a la historia surgió por el interés del Departamento de Defensa de EE.UU. que funda una agencia de investigación avanzada, ARPA en 1958 para motivar a los investigadores universitarios para superar en tecnología militar a Rusia.

Así surge ARPANET una red de ordenadores cuyo primer director era psicólogo interesado en la informática interactiva. Se basó en una revolucionaria tecnología de transmisión de telecomunicaciones de un californiano y un inglés. Esta tecnología pretendía una red de comunicaciones flexible y descentralizada para construir un sistema de comunicaciones militar. Se dispersaron los nodos entre departamentos de distintas universidades norteamericanas. ARPANET lo diseñó una empresa de ingeniería acústica fundada por profesores del MIT (Universidad Tecnológica de Boston) en el que trabajan también científicos e ingenieros de Harvard.

El siguiente paso fue conectar ARPANET con otras redes de ordenadores. Dos informáticos de Standford, Kahn y Cerf elaboraron el proyecto básico de la arquitectura de Internet. Se dieron cuenta que era necesario unos protocolos de comunicación estandarizados. En 1978 lo consiguieron Cerf, Postel y Cohen en la Universidad de California del Sur. Fue transferido ARPANET a la Agencia de Comunicación de la Defensa.

El Departamento de Defensa preocupado por la seguridad creó una red interna, la red MILNET. ARPA-INTERNET se destinó a la investigación. En 1984 la Fundación Nacional para la Ciencia estableció su propia red informática de comunicaciones y utilizó ARPA-INTERNET como eje troncal. En 1990, el Pentágono encargó la gestión del proyecto a la Fundación para la Ciencia que poco después permitió la privatización. Se pudo ampliar la red gracias a la incorporación de nuevos nodos y muchas reconfiguraciones.

Otra influencia que tuvo Internet es la tradición de interconexión informática autónoma y alternativa. Entre ellos la corriente de los Tablones de Anuncios, el diseño de 
un programa MODEM y un programa de ordenador FIDO, la red experimental BITNET o el sistema UNIX de los laboratorios Bell. Hasta que estudiantes de la Universidad de Carolina del Norte diseñaron un programa de comunicación entre ordenadores UNIX y en la Universidad de Berkeley, nodo de ARPANET diseñó un programa para crear un puente entre las dos redes. Estas redes terminaron uniéndose para formar Internet.

Otro de los avances fue permitir el acceso abierto a toda la información existente sobre sistemas de software y la influencia de la cultura hacker. Bajo esta filosofía se creo el Fundación para el software libre o el sistema operativo LINUX donde los usuarios podían perfeccionarlo y publicar sus modificaciones.

La aplicación de la World Wide Web de Tim Berners-Lee consiguió que se pudiera compartir la información desarrollada por este programador inglés que perfeccionó su programa Enquire. Este software permite sacar e introducir información de y en cualquier ordenador conectado a Internet (HTTP, HTML y URL). En colaboración con otro programador construyó un programa navegador con sistema de hipertexto.

Cuando el CERN publicó la red de programa para WWW otros diseñadores se inventaron más software similar. Netscape Communications puso en la red el primer navegador comercial el Netscape Navigator. Tras el éxito de Navigator, Microsoft descubrió Internet e incluyó junto a su software Windows $95 \mathrm{su}$ propio navegador Internet Explorer.

Así en 1995 nace Internet cuando estaba privatizado y su arquitectura técnica abierta permitía la conexión a todas las redes informáticas de forma universal, y la red podía funcionar con un software adecuado pues existían varios navegadores fáciles de usar.

La política estratégica militar del Departamento de Defensa apoyar con finanzas y dejar trabajar a los científicos de forma autónoma se mostró como la más inteligente y la que permitió que adquiriera ciertos rasgos: una estructura reticular, un poder de computación distribuido entre los diversos nodos y una redundancia de funciones en la red.

Los científicos contaban con el objetivo de optimizar el uso de costosos recursos informáticos a base de compartir tiempo on-line. Eso fue el principio del correo electrónico, que es hoy en día la aplicación más popular en Internet.

El ambiente en el que se creó la Red era relajado, fuera de miras militares sino de mejora del mundo mediante la comunicación entre ordenadores. ARPA estuvo formada por científicos universitarios en la que supieron unir fuerzas humanas. 
En los años 80 fue evidente que EE.UU. era superior en tecnología a Rusia y este factor fue uno de los desencadenantes de desintegración del Imperio soviético y la época de la perestroika de Gorbachov.

La Red no se originó en el mundo empresarial, sino que fue una labor de sinergias positivas entre instituciones gubernamentales, grandes universidades y centros de investigación. Hubo ocasiones en las que tanto compañías privadas (Bell) como instancias públicas (Oficina General de Correos) no impulsaron el proyecto que pasó a otras manos.

Las universidades jugaron un papel fundamental en su apoyo a las redes comunitarias de base pues contaban con máquinas de mayor potencia y contribuyeron a que la Red alcanzara pronto a todo el mundo.

Los estudiantes que contribuyeron al avance de la Red mantenían una filosofía en la que primaba la libertad individual, el pensamiento independiente y el afán solidario de compartir y colaborar con otros investigadores. Entre los miembros más revolucionarios, en cuanto a política, se mantenía que la Red podría ser una herramienta de liberación que transmitiría a la gente el poder de la información frente a empresas o gobiernos.

En los años 80 las empresas de informática y de telecomunicaciones pusieron en marcha servicios de correo electrónico y Redes de Área Amplia.

El control del gobierno norteamericano sobre ARPA-INTERNET suponía un inconveniente para la conexión con redes internacionales. La Fundación para la Ciencia permitió el acceso de NSFNET y su privatización en 1995, hecho que permitió la proporción de redes no estadounidenses y su difusión global.

La transparencia, la flexibilidad de la Red permitió que ejes troncales como ARPANET se conectaran con Redes de Área Local. La estructura abierta de Internet procuró un acuerdo sobre un estándar internacional común. Los estándares TCP/IP de ARPANET se mostraron flexibles para integrar diversos sistemas de conexión y de esa manera prevaleció. Hubo otras redes públicas de telecomunicaciones y algunas redes comerciales que adoptaron protocolos X.25 que son compatibles con ARPANET.

Este carácter abierto de la Red permitió beneficiarse de las colaboraciones de las iniciativas privadas para componerla como fue el caso de Tim Berners-Lee que se vio apoyado a su vez por la comunidad de internautas y hackers.

Internet sigue creciendo debido a tres rasgos: por el carácter descentralizado de la Red, por los protocolos abiertos y por las instituciones que gestionan Internet que mantienen como rasgos la transparencia y la cooperación. 
ARPA desde un principio ejerció una autoridad benevolente sobre la Red. Posteriormente se estableció un Comité de Actividades de Internet (IAB) que aunaba los expertos principales de las instituciones que habían creado ARPANET pero abierto a otros expertos internacionales. La IAB se dividió posteriormente en dos organizaciones IEFT y IRFT.

En enero de 1992 se formó la Sociedad Internet dirigida por los especialistas Cerf y Kahn. Se trataba de una ONG que supervisaba ambas organizaciones. Aseguró la participación internacional aunque se vio amenazada por Europa.

Se formó después para la supervisión IANA pero a la muerte de su director, Postel, se planteó el proyecto del difunto ICANN, una corporación que está formada por el conjunto de los miembros y tres organizaciones de apoyo.

ICANN ha conseguido mantener la transparencia y la abertura, pues admite a cualquier persona con conocimientos técnicos para asegurar la representación mundial. Muchos gobiernos, sin embargo, acusan a ICANN de pertenecer al dominio norteamericano.

Existe el Consorcio World Wide Web, una organización internacional abierta que depende del MIT en EE.UU. y en Europa del instituto francés INRIA, dirigido por Tim Berners-Lee. Se ha conseguido un relativo equilibrio en la gestión de la Red, sin otras fuerzas de poder que lo pervierta.

\subsection{Estructura}

Entre el primer título una visión panorámica y el segundo título hay una relación de contenidos que no presenta bien en el segundo título, pues utiliza la información anterior para añadir un comentario específico sobre la intencionalidad de los actores involucrados en el proceso de Internet.

Repite algunas ideas que quiere destacar como la unión de fuerzas del título: la ciencia, la investigación militar y la cultura de la libertad (página 8).

Hay palabras técnicas que no define y traduce directamente del inglés como “comunicación de paquetes" (página 1 y 9), “think-tank" (en la página 1 por detrás). Hay algunos conceptos que se entienden porque da una explicación más adelante (por ejemplo, "eje troncal" en la página 3). Esta poca claridad semántica, en ocasiones, dificulta la lectura de este artículo. Otro elemento que entorpece es la aparición de muchas siglas a lo largo del texto. 


\section{CONCLUSIONES}

El primer artículo procura una panorámica de futuro del uso de Internet y de las posibilidades que ofrece, que se multiplicará gracias a una mentalidad más tecnológica por parte de los profesionales involucrados en la Red. La hipertextualidad procura una mayor rapidez del acceso a la información y contribuye a una visión global mayor y personalizada del tema o servicio buscado. La interactividad da un papel participativo mayor al ciudadano, y procura una mentalidad más proactiva.

El segundo artículo narra la historia de casualidades del Departamento de Defensa norteamericano para la creación de Internet, cuando es actualmente una herramienta puesta al servicio de cualquier ciudadano para acceder a la información y otros servicios, y con el que accede a una ciudadanía global.

\section{BIBLIOGRAFÍA RECOMENDADA}

- Alonso, J. (2006). “Comunicación virtual: elementos y dinámicas”, Gestión Editorial, Visionnet.

- Alonso, J. y Martínez, L. (2003). “Medios interactivos en Internet: caracterización y contenidos", en Salaverría, R. y Díaz Noci, J. Manual de Ciberredacción periodística. Barcelona: Ariel.

- Berners-Lee, T., (2000), Tejiendo la Red, editorial siglo XXI España editores, Madrid.

- Castells, M. (1996): La era de la Información. Economía, Sociedad y Cultura. VOL 1. La Sociedad Red. Madrid, Alianza Editorial.

- Castells, M., (2001): La galaxia Internet. Madrid: Plaza \& Janés Editores.

- Codina, L1. y Marcos, M.C. (2005): "Posicionamiento Web: conceptos y herramientas", en El profesional de la Información, v.14, n 2, marzo-abril 2005, pp. 84-99. Disponible en Internet: http://elprofesionaldelainformacion.metapress.com/media/cbplvltxqmt6fcxwhk dr/contributions/w/m/h/m/wmhmdy4ex9864u42.pdf

- Cornella, A., (2002), Hacia la infonomía.com. La empresa es información, Ediciones Deusto, Barcelona. 
- García de León y Garrido (2002): “los sitios web como estructuras de información: un primer abordaje en los criterios de calidad", en Biblios, $\mathrm{n}^{\circ} 12$. Disponible en Internet: [http:/ / eprints.rclis.org/archive/00002352/01/B12-02.pdf]

- Goto, K. y Cotler, E. (2002) Rediseño de sitios web, Prentice Hall, Madrid

- Hassan, Y. y Martín, F.J. (2003). “Guía de Evaluación Heurística de sitios web”, en No Solo Usabilidad journal, n 2.30 marzo de 2003.

- López García, G. (2005): Modelos de comunicación en internet. Valencia, Tirant Lo Blanch.

- Marcos, M.C. (2007): "La usabilidad en España, un boom muy necesario", en Anuario ThinkEPI 2007. Análisis de tendencias en información y documentación, pp. 178-183. Disponible en Internet: [http://www.mcmarcos.com/pdf/2007Anuario-ThinkEPI-MC-Marcos-Usabilidad-Espana.pdf]

- Marcos M.C. y Rovira C (2006): “Las webs parlamentarias: funciones y elementos de su interfaz en el acceso a la información", en Rev. Esp. Doc. Cient, 29, I, pp. 11-26

- Maestros del Web: Dossier de Análisis de un sitio Web en [http://www.maestrosdelweb.com/editorial/analisisweb]

- Monistrol, R., Rovira, C. y Codina, Ll. (2006) "Sitios Web de museos de Cataluña: Análisis y propuesta de evaluación", en Hipertext.net, núm. 4. [http: //www.hipertext.net]

- Nielsen, J. (2000) Usabilidad, diseño de sitios web, Prentis Hall, Madrid

- Rojas, O. I. y otros, (2005), Blogs: la conversación en Internet que está revolucionando medios, empresas y a ciudadanos, ESIC editorial, Madrid.

- Salaverría, R., (2005), Redacción periodística en Internet, Eunsa, Pamplona. 